\title{
The Role of Neurostimulation in the Treatment of Epilep- sy-A Commentary on the State of Its Use and Its Potential Worldwide
}

\author{
Arun Swaminathan, MD* \\ Assistant Professor of Neurology, University of Nebraska Medical Center, Omaha, NE, USA
}

*Corresponding author: Arun Swaminathan, MD, Assistant Professor of Neurology, University of Nebraska Medical Center, Omaha, NE, USA

Epilepsy is a chronic condition that affects $1-3 \%$ of the population world-wide [1]. There are 50-65 million people worldwide with epilepsy [2]. Approximately twothirds of the epilepsy population is well controlled on about seizure medications alone, while the remaining one-third remains refractory with poor or insufficient seizure control on two or more medications [3]. These refractory patients are often in desperate need of additional therapies besides medications for seizure management. Epilepsy surgery, trial medications, ketogenic diet are some other options, which have shown promise in a good number of patients. Neurostimulation joins the list of available treatments that have helped transform the landscape of treatment of refractory epilepsy.

Vagal nerve stimulation (VNS) represents the earliest and longest used method of neuromodulation for epilepsy. Implanted and transdermal VNS approaches have both been used and the implanted technique has been proven to be more superior and has become the procedure of choice. VNS implants offer a good treatment option for generalized, multifocal and inoperable epilepsies and are often preferred by patients who wish to have the least invasive option used in them. The VNS also offers relatively good results in patients with focal epilepsies that may be unable or unwilling to undergo more invasive surgical treatments. VNS implantations have been used in Europe since 1994 and the USA since 1997 with excellent results and rare complications in a large cohort consisting of more than 100,000 patients. Cost of implantation and up keep of the device, including surgeries for revision or battery replacement, are low and make this a very attractive option. The newly introduced cardiac monitoring feature with responsive changes on the VNS have increased its potential benefits to include lowering the risk of sudden death in epilepsy (SUDEP) even further. Unfortunately, many parts of the world outside of Europe and the USA do not offer this option regularly and its use is highly uncommon. Transdermal VNS remains a less effective option and is usually utilized as a research tool only. Improving availability and access to the use of this device would help combat the burden of epilepsy worldwide as the costs are quite reasonable and the benefits remain manifold. Potential downsides to the use of the VNS device include its nature as a palliative rather than a curative option, lack of significant response $(<50 \%$ seizure reduction) in a reasonably higher number of patients (around $30-40 \%)$ and the need to for multiple visits to the physician making it challenging for patients and families or caretakers. Added benefits also include its use for the treatment of depression, for which it was approved by the FDA in 1999, which makes it a very attractive option for multimodal benefit in many epilepsy patients with coexistent depression, who make up a large fraction of the epilepsy population.

Responsive neurostimulation (RNS) represents a newer advancement in the field of neuromodulation for epilepsy, with complete FDA approval given in 2013 after initial trial onset in 2010-2011. The benefits of closed loop responsive stimulation for epilepsy have been studied in the last few years and significant improvements have been noted in seizure control

Citation: Swaminathan A (2020) The Role of Neurostimulation in the Treatment of Epilepsy-A Commentary on the State of Its Use and Its Potential Worldwide. Int J Neurol Neurother 7:097. doi. org/10.23937/2378-3001/1410097

Accepted: February 20, 2020: Published: February 22, 2020

Copyright: (C) 2020 Swaminathan A. This is an open-access article distributed under the terms of the Creative Commons Attribution License, which permits unrestricted use, distribution, and reproduction in any medium, provided the original author and source are credited. 
with the use of this device. Chronic electrocorticography with closer monitoring and resultant changes in treatment also make this a very attractive option, especially in patients for whom resection of eloquent cortex is not an option. The RNS has been found to be the treatment of choice in epilepsy patients with bitemporal involvement, eloquent cortical seizure onset such as motor, language or visual cortex or large networks with multifocal or diffuse involvement, due to its network modulating properties. Improvement in detection and responsive algorithms has resulted in improved detection and treatment rates producing better seizure outcomes over the last few years. While not guaranteeing seizure freedom, it does offer substantial seizure reduction in many patients with an improvement in cognitive and quality of life measures as well. The restrictive cost of implanting and maintaining the RNS device does make it a less attractive option in many parts of the world and its current usage is restricted to the United States only. Producing a cheaper version or a less complicated version of the device with more simplistic algorithms may result in the creation of a device that may offer benefit for a large cohort of patients worldwide at the expense of a certain degree of sophistication or accuracy. Drawbacks of using this device include its highly invasive nature, exorbitant cost of implantation and maintenance, need for high quality technical support and assistance, potential risks for infection and hemorrhage and the need for a high level of technical, radiological and surgical accuracy to ensure proper and effective implantation. The device has however, continued to show increasing popularity with about 1800 patients implanted in the USA thus far with an expected large increase in numbers over the next few years. Ancillary benefits also include decrease in anxiety and depression, similar to the VNS device, although there is no specific FDA approval in place for such an indication. The RNS has been found to show good results in patients with a previously implanted VNS device as well, reflecting their combined effects on the epileptic networks of the brain.

Deep brain stimulation (DBS) represents the latest modality in the field of neuromodulation for epilepsy. While the technology itself is not entirely new, in that it has been used for the treatment of Parkinson's disease and tremor for upwards of 20 years, its application to the field of epilepsy is relatively novel. Initial trials for DBS started in 2010 (SANTE), before the FDA finally gave approval in 2018, after years of analyzing SANTE follow up data. The anterior nucleus of the thalamus represents the most common target for the treatment of epilepsy while alternative approaches have included targeting the dorsomedial and centromedian nuclei, the cerebellum or the hippocampi for certain subtypes of epilepsy. While availability of technique and knowledge of the procedure are present worldwide, expertise in performing it and costs of surgery may prove to be an obstacle. DBS still represents a very reasonable approach to the management of refractory epilepsy with good applicability to a variety of epilepsy syndromes. Multifocal or generalized epilepsies would theoretically represent the best patient types for a DBS implantation, although focal epilepsies with rapid generalization of seizures may be fairly good candidates as well. DBS probably represents a network effect of neuromodulation, rather than a targeted treatment like RNS, and certainly has widespread applicability in terms of choice of patients to perform the procedure in. Potential drawbacks include the degree of invasiveness, difficulty with finding the desired targets, incidence and risk of intracranial hemorrhage and infection as well, while the cost of imaging, evaluation and surgical implantation always remain prohibitive in many countries. DBS has shown great promise in many conditions besides epilepsy and movements disorders (Parkinson's, dystonia and tremor), such as anxiety, depression, OCD, anorexia, addiction and Alzheimer's disease [4]. The DBS was used most recently in Canada to treat a case of alcohol dependence and Chinese centers are performing it experimentally for its use to treat opioid dependence with targeting involving the nucleus accumbens and anterior limb of the internal capsule, with promising results in the early stages [5]. More than 160,000 patients have been implanted with DBS worldwide, across all indications, with good safety profiles being achieved. Most of these patients have been implanted for Parkinson's or tremor with current literature reflecting that about 825 patients having being implanted with DBS for epilepsy cross a variety of targets, with the anterior thalamic nucleus being the most popular site.

Neuromodulation as a whole has taken giant steps forward over the last few years due to the change in approach to the treatment of epilepsy as a network disorder rather than an isolated seizure focus with wide ranging effects. Seizure freedom or significant reduction have always represented the primary objectives of neuromodulation and the devices mentioned above achieve fairly good rates of seizure freedom ranging from $50-75 \%$ over a few years [6]. Secondary benefits include improvements in cognition, reduction in depression, anxiety and similar psychiatric disturbances and minimal to substantial improvements in quality of life, depending on patient choice and seizure outcomes. Further uses for neuromodulation continue to be explored, especially in the fields of psychiatry and addiction medicine, in addition to the expanding role of neuromodulation for epilepsy and other neurological disorders.

Other non-invasive approaches involving transcranial magnetic stimulation (TMS), non-invasive VNS, transcranial direct nerve stimulation (tDCS) and trigeminal nerve stimulation (TNS), among others, are also being investigated but would need more data 
and greater numbers to determine efficacy and applicability of use worldwide. The numbers available for these modalities are quite small with initial results being unimpressive with less than $30 \%$ seizure reduction seen in most case series. Improved techniques or stimulation parameters may offer better results in future, but the current interest in these devices is dwindling, especially since the previously mentioned options are quite popular and reasonably effective.

Cost and availability of surgical equipment and techniques currently represent the greatest obstacles in widespread use of these techniques, but these can be overcome with concentrated efforts targeting financial and infrastructural investment and awareness towards the field of epilepsy as a whole. Neuromodulation is a relatively nascent field with many advances expected in this field over the next few years, resulting in many significant improvements and modifications to current treatment protocols over time. These changes are expected to benefit scientific knowledge, medical practice, patients, and humanity as a whole and offer neurostimulation a unique and special place in the lineup of epilepsy treatments and scientific developments of the medical profession.

\section{References}

1. CDC Fast facts 2015.

2. WHO Global burden of assessment of disease. 2015-2018.

3. Kwan, Brodie (2000) Early Identification of Refractory Epilepsy. NEJM.

4. Lozano, Lipsman N, Bergman H, Brown P, Chabardes S, et al. (2019) Deep Brain Stimulation: Current Challenges and Future Directions. Nat Rev Neurol 15: 148-160.

5. Qu, Ge S, Li N, Wang W, Yang K, et al. (2019) Clinical Evaluation of Deep Brain Stimulation of Nucleus Accumbens/ Anterior Limb of Internal Capsule for Opioid Relapse Prevention: Protocol of a Multicentre, Prospective and Double-Blinded Study. BMJ Open 9.

6. Boon P, De Cock E, Mertens A, Trinka E (2018) Neurostimulation for Drug-Resistant Epilepsy: A Systematic Review of Clinical Evidence for Efficacy, Safety, Contraindications and Predictors for Response. Curr Opin Neurol 31: 198210. 